\title{
Incidência de Neoplasia Intra-Epitelial Cervical Registrada no Posto Central de Tangará da Serra-MT no Período de 2009 a 2011
}

\section{Incidence of Cervical Intraepithelial Neoplasia in Central Public Station of Tangará da Serra- MT from 2009 to 2011}

\author{
Aniely Loiza Pedrozo; ; Loriane Tagliari Medina ${ }^{\text {a* }}$ \\ âniversidade de Cuiabá, Curso de Farmácia, MT, Brasil \\ *E-mail: lorianetagliari@hotmail.com \\ Recebido: 11 de abril de 2014; Aceito: 20 de outubro de 2014
}

\begin{abstract}
Resumo
O interesse pelo Papilomavírus Humano surgiu devido à associação do vírus com o câncer cervical. Atualmente, consiste em um grave problema de saúde pública por ser uma das principais causas de mortalidade mundial. O objetivo do presente trabalho é quantificar os números de casos de neoplasia intra-epitelial cervical registrados no Posto Central, entre os anos de 2009 a 2011, em mulheres a partir dos 18 anos, moradoras do município de Tangará da Serra-MT. As informações foram obtidas no banco de dados implantado no Posto Central de Tangará da Serra-MT, o SISCOLO (Sistema de Informação do Câncer do Colo do Útero). A amostra foi constituída de 28 mulheres, obedecendo aos critérios propostos de inclusão e exclusão. Após a análise dos dados, verificou-se que, no ano de 2009, quinze mulheres apresentavam neoplasia intra-epitelial cervical (NIC), 53\% caracterizada por NIC III, 20\% NIC I e 27\% NIC II. Em 2010, metade da amostra apresentava NIC II e a outra metade NIC III. Já em 2011, existiam registros de 7 mulheres com neoplasia intra-epitelial cervical, sendo que NIC II e NIC III apresentaram distribuição semelhante e NIC I apresentou menor incidência. Conclui-se que a incidência de neoplasia intra-epitelial cervical em mulheres de Tangará da Serra-MT tem diminuído com o passar dos anos, não mostrando variação na idade das mulheres afetadas pelas lesões, que se encontram dentro do grupo de adulta-idosa. Ressalta-se a importância da detecção precoce das lesões precursoras da cérvice, uma vez que, quando diagnosticadas nos estágios iniciais das neoplasias intra-epiteliais cervicais, aumentam-se as possibilidades de cura e evita-se a progressão da lesão para carcinoma invasivo.
\end{abstract}

Palavras-chave: Epidemiologia. Doenças Sexualmente Transmissíveis. Saúde da Mulher.

\begin{abstract}
The interest for Human Papilloma virus emerged due to its association with cervical cancer. Currently, it is considered a serious public health problem, once it is a major cause of mortality worldwide. The objective of this study is to quantify the number of cases of cervical intraepithelial neoplasia in 18 years or older women, from a Local Health Unit in the city of Tangara da Serra -MT, from 2009 to 2011. Information was provided from the health center database, the SISCOLO (Information System on Cervical Cancer). The sample consisted of 28 women according to the inclusion and exclusion criteria. After data analysis, it was found that, in 2009, 15 women had cervical intraepithelial neoplasia (CIN), 53\% characterized by CIN III, 20\% by CIN I, and 27\% by CIN II. In 2010, half of the sample had CIN II, while the other half had CIN III. In 2011, 7 women with cervical intraepithelial neoplasia were registered, with similar distribution for CIN II and CIN III, and a lower incidence of CIN I. It was concluded that the incidence of cervical intraepithelial neoplasia in women from Tangara da Serra-MT has decreased over the years, with no variation in age of women affected by lesions that are within the adult-elderly group. It is worth emphasizing the importance of early detection of precursor lesions of the cervix, since the chances of cure are greater when diagnosed in the early stages, preventing lesion progression to invasive carcinoma.
\end{abstract}

Keywords: Epidemiology. Sexually Transmitted Diseases. Women's Health.

\section{Introdução}

A descoberta e o tratamento de verrugas genitais são relatados desde a antiguidade sendo que, inclusive, Galeno (129-199 d. C) fez inscrições sobre práticas populares para eliminar verrugas. Além disso, ainda na História antiga, os médicos gregos e romanos foram os primeiros a constatar a transmissibilidade sexual dessas verrugas. No entanto, a presença de lesões verrucosas estava correlacionada ao homossexualismo ou falta de higiene íntima ${ }^{1-3}$. Contudo, apenas no final do século XIX descobriu-se a natureza infecciosa dessas verrugas através da auto inoculação realizada pela primeira vez em Londres, no ano de 1891, por Joseph Payne. Posteriormente, no início do século XXI,
Ciuffo, suspeitando da causa viral das verrugas, realizou um experimento utilizando filtrado e se auto-inoculou para produzir verrugas nas mãos. Entretanto, somente em 1950, conseguiu-se identificar o Papilomavírus Humano - HPV como agente causal das verrugas ${ }^{4,5}$.

Apesar dos estudos acima citados, pode-se afirmar que o interesse maior pelo HPV surgiu na década de 70 , devido à sugestão de Zur Hausen sobre a associação do vírus com o câncer cervical. Essa associação foi confirmada após vários estudos moleculares, epidemiológicos e clínico-patológicos que evidenciaram a presença do HPV no câncer cervical e nas lesões precursoras ${ }^{6-8}$. As lesões precursoras na cérvice podem ser classificadas como neoplasia intra-epitelial 
cervical (NIC). Em Bethesda, 1988, essas lesões de colo uterino passaram a ser classificadas de acordo com suas características cito-histopatológicas: NIC I, displasia leve (lesão de baixo grau), NIC II ou displasia moderada, e NIC III ou displasia grave (lesões de alto grau) ${ }^{9-12}$. Como o câncer de colo de útero é precedido por essas lesões precursoras da cérvice, a identificação, nessas fases de evolução, pode ser facilmente tratável, antes que a lesão se torne invasiva.

Dessa forma, sendo a patogenia do trato genital inferior mais prevalente entre as mulheres sexualmente ativas, independente do estágio de NIC, e, principalmente, devido as evoluções da biologia molecular, o HPV tem sido foco principal de estudos que visam determinar seu papel na carcinogênese do colo uterino ${ }^{11,13,14}$.

Entretanto, a ocorrência de eventos carcinogênicos não é resultado de um único e isolado acidente, mas pelo contrário, ocorre devido uma série de eventos independentes e com efeitos cumulativos. Por isso, a ocorrência de lesões e até mesmo do carcinoma uterino dependerá do tipo de HPV envolvido, da carga viral e da persistência e integração com a célula hospedeira, de fatores imunológicos inerentes ao hospedeiro do vírus e de fatores ambientais ${ }^{15,16}$.

Uma em cada cinco mulheres no mundo é portadora do HPV e a cada ano há registros de 137 mil casos novos no Brasil. Sendo assim, o Brasil apresenta-se como um dos líderes mundiais em incidência de HPV sendo que a infecção possui como alvo mais frequente mulheres entre 15 e 25 $\operatorname{anos}^{17-19}$. Entretanto, pouco se sabe sobre o número de casos no interior de Mato Grosso.

A estrutura de atendimento para pacientes com neoplasia intra-epitelial cervical - NIC associada ou não com a infecção pelo Papilomavírus Humano - HPV é constituída pelo Posto Central do município de Tangará da Serra-MT, local de acolhimento das portadoras de displasias cervicais, em conjunto com o CTA-SAE (Centro de Testagem e Aconselhamento-Serviço de Assistência Especializada), responsável pelo diagnóstico e tratamento das lesões. Estas estruturas de atendimento especializado realizam todo o acompanhamento necessário da paciente com relação a terapêutica empregada, promovendo o uso racional dos medicamentos, e garantindo o cumprimento da terapêutica prescrita ao paciente ${ }^{1}$. Fato esse de extrema importância para que este trabalho seja realizado com a garantia de resultados fidedignos.

Portanto, o objetivo do presente trabalho é quantificar os números de casos de neoplasia intra-epitelial cervical registrados no Posto Central, entre os anos de 2009 a 2011, em mulheres a partir dos 18 anos, moradoras do município de Tangará da Serra-MT.

\section{Material e Métodos}

O presente estudo teve a aprovação da Comissão de Ética em Pesquisa com protocolo $n^{\circ}$ 2010-227, da Universidade de Cuiabá - Unic.
Realizou-se pesquisa no Posto Central, centro de referência no tratamento de afecções do colo uterino em Tangará da Serra-MT para quantificar os casos de displasia originados em pacientes acima de 18 anos, residentes neste município, entre 2009 a 2011.

O método de coleta dos dados ocorreu através da análise do banco de dados implantado neste centro especializado, o SISCOLO - Sistema de Informação do Câncer do Colo do Útero.

Os critérios de inclusão foram mulheres maiores de 18 anos que apresentavam neoplasia intra-epitelial cervical, atendidas pelo Posto Central e CTA-SAE do município de Tangará da Serra-MT. Os critérios para exclusão de pacientes foram: mulheres que não apresentavam neoplasia intraepitelial cervical, idade inferior a 18 anos e pacientes que não eram acompanhadas pelo Posto Central e/ou CTA-SAE.

Dessa forma, a amostra do presente estudo foi constituída por mulheres portadoras da infecção por HPV e que desenvolveram neoplasia intra-epitelial cervical devido a esta infecção prévia. Por fim, os dados obtidos foram analisados estatisticamente por frequência relativa e frequência absoluta.

\section{Resultados e Discussão}

Após a análise do banco de dados, SISCOLO, verificouse que, da amostra analisada (28 mulheres), a faixa etária de maior incidência de neoplasia intra-epitelial cervical está entre 30 a 39 e 50 a 59 anos (Quadro 1). No entanto, quando comparamos o ano com a idade acometida, percebemos que o maior número de casos foi observado em 2009, com 15 mulheres, apresentando de 23 a 56 anos. No ano subsequente, apenas 6 casos foram observados e a idade das mulheres variava de 25 a 54 anos. Já em 2011, apesar de apenas 7 casos terem sido notificados, a média de idade das mulheres acometidas aumentou para 45 anos.

Quadro 1: Incidência de mulheres com neoplasia intra-epitelial cervical distribuída por idade, nos anos de 2009 a 2011

\begin{tabular}{|c|c|}
\hline Incidência por Idade & Número de Casos \\
\hline 18 a 29 anos & 5 casos \\
\hline 30 a 39 anos & 9 casos \\
\hline 40 a 49 anos & 3 casos \\
\hline 50 a 59 anos & 9 casos \\
\hline 60 a 69 anos & 2 casos \\
\hline
\end{tabular}

Fonte: Dados da pesquisa.

Os dados sobre a variação de idade do presente estudo corroboram com os fornecidos pelo INCA, nos quais a manifestação de câncer de colo uterino é evidenciada na faixa etária de 20 a 29 anos, sendo que aumenta seu risco até atingir seu pico entre 50 a 60 anos. Além disso, na região CentroOeste juntamente com o Nordeste do Brasil, o câncer de colo uterino ocupa a segunda colocação em incidência. Logo, esse 
perfil da população analisada apresenta correlação com o perfil estudado, entre 2008 e 2009, na cidade de Anápolis-Goiás. Os autores mostram que a faixa etária variou de mulheres maiores de 19 anos até $79 \operatorname{anos}^{20,21}$.

Os resultados obtidos nesse estudo não se assemelham com o perfil da população de um estudo realizado em MaringáParaná, onde a idade mínima observada pelos autores foi de 15 anos e máxima de 52 anos. No entanto, corroboram com estudo realizado em Cacoal-Rondônia, entre 2005 e 2007, onde a faixa etária acometida por NIC II, NIC III e carcinoma in situ é de 16 a 79 anos, evidenciando uma larga faixa etária acometida com tais lesões.

Uma vez que a infecção por HPV está intimamente relacionada com as neoplasias intraepiteliais cervicais e com o câncer de colo de útero, existe uma grande preocupação com as altas taxas dessa infecção viral. O Brasil possui taxas de infecção pelo HPV de 17,8\% a 27\%, com prevalência maior em mulheres abaixo dos $35 \operatorname{anos}^{22-25}$. Esses dados divergem dos obtidos nesse estudo, em que a maior incidência das lesões foram observadas na faixa etária de 30 a 39 e de 50 a 59 anos, caracterizando o diagnóstico das lesões em mulheres com idade mais avançada que a média nacional.

Considerando a faixa etária com maior predomínio de NIC, entre 30 a 39 e 50 a 59 anos, dados recentes concordam com o presente estudo, como o trabalho realizado na Unidade de Alta Complexidade de Oncologia, em Roraima, que teve, como faixa etária dominante, pacientes de 30 a 60 anos $^{26}$.

No entanto, o estudo feito na cidade de Anápolis-Goiás relatou que a faixa etária com maior incidência de neoplasia intra-epitelial cervical é entre 40 a 49 anos, incidência esta que não foi encontrada em Tangará da Serra-MT. Já estudo realizado em 20 aldeias do Parque do Xingu, constatou que as faixas etárias de maior prevalência de NIC em mulheres indígenas são dos 25 aos $29 \operatorname{anos}$ e dos 40 aos 44 anos. Embora não possa ser comparado diretamente com os dados desse estudo por ter sido avaliado em indígenas, trata-se de um dos poucos trabalhos da região de Mato Grosso ${ }^{21,27}$.

Nesse sentido, outro estudo que discorda com a faixa etária encontrada nesse presente trabalho é o realizado no estado do Rio Grande do Norte, no qual a maior distribuição de neoplasia intra-epitelial celular foi encontrada na faixa etária de 20 a 49 anos. Todas estas informações ressaltam a importância da realização do rastreamento do câncer de colo uterino em todas as mulheres sexualmente ativas, independentemente da idade ${ }^{28}$. Sendo assim, através da análise do perfil da população estudada constatou-se que a incidência de NIC por Papilomavírus Humano em Tangará da SerraMT é maior em mulheres adultas, com faixa etária acima de 30 anos, indicando que as mulheres adultas jovens possam estar mais conscientizadas com os métodos de prevenção de saúde, facilidade e rapidez no atendimento prestado pela rede pública e/ou, talvez, pelo fato das mulheres mais maduras não possuírem o hábito de utilizar método preservativo de barreira e de não procurar o serviço de saúde para realizar os exames preventivos, acreditando estarem protegidas por um relacionamento estável.

Com relação à idade máxima na qual se encontrou NIC em Tangará da Serra, 68 anos, sugere-se a realização do exame citopatológico anualmente, mesmo a mulher não estando em fase reprodutiva. Contudo, esse dado discorda da literatura já descrita, pois, de acordo com INCA, a máxima incidência de câncer de colo uterino está entre 50 e 60 anos, sugerindo que mais estudos devem ser realizados em mulheres idosas ${ }^{20}$.

Quanto à quantidade de casos de neoplasia intra-epitelial cervical, distribuídas segundo o tipo de NIC e de acordo com o ano, foi possível constatar uma variação na frequência de casos de NIC. No ano de 2009, verificou-se a incidência maior de NIC III (53\%) e a distribuição de NIC I e NIC II apresentou valor aproximado $20 \%$ e $27 \%$, respectivamente. Em 2010, foram registrados 6 casos de neoplasia intraepitelial cervical, dos quais a metade foi diagnostica com NIC II (50\%) e a outra metade como NIC III. No ano de 2011 registrou-se 7 casos de neoplasia intra-epitelial cervical, sendo que NIC II e NIC III apresentaram distribuição semelhante $(43 \%)$ e NIC I apresentou a menor incidência (14\%) (Figura 1 e 2). Através desse estudo, pode-se verificar que há maior prevalência de lesões intra-epiteliais cervicais de alto grau, ou seja, NIC II e III.

Figura 1: Incidência de neoplasia intra-epitelial cervical, em mulheres atendidas pelo Posto Central de Tangará da Serra-MT, no ano de 2009.

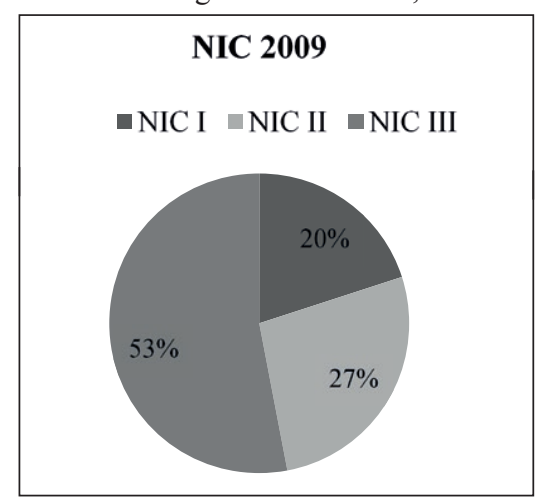

Figura 2: Incidência de neoplasia intra-epitelial cervical, em mulheres atendidas pelo Posto Central de Tangará da Serra-MT, no ano de 2011.

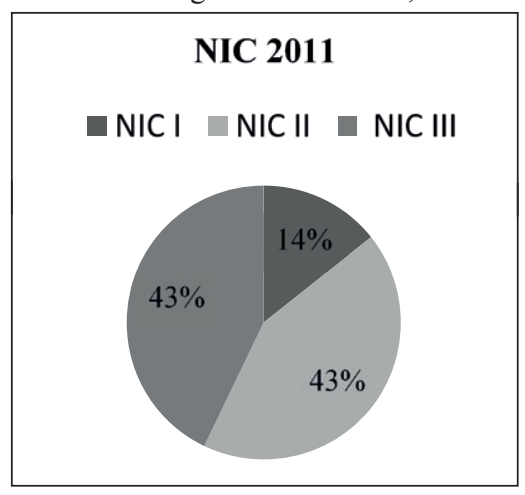


Na presente pesquisa, verificou-se maior incidência de lesões intra-epiteliais cervicais de alto grau, ou seja, NIC II e III. Dessa maneira, os resultados se assemelham com os dados obtidos em Maceió-Alagoas, no qual 73,3 \% da população do estudo apresentaram lesão intra-epitelial de alto grau e $26,7 \%$ possuíam lesão de baixo grau ${ }^{29}$.

De posse desses dados, pode-se constatar com esse estudo uma redução na incidência de neoplasia intra-epitelial cervical com o passar dos anos, porém, verifica-se uma maior ocorrência de NIC de alto grau, o que caracteriza um agravamento do quadro clínico da paciente. A redução no número de casos novos de NIC em mulheres acompanhadas pelo Posto Central corrobora com o estudo realizado em indígenas na aldeia do Xingu, entre 1989 a 1996, que constatou a reduzida incidência de neoplasia intra-epitelial cervical, com registro de 3 casos de NIC I, 1 caso de NIC II e 1 caso de NIC III ${ }^{27}$.

Essa redução no número de displasias pode ser atribuída a uma maior informação transmitida para as mulheres sobre a importância do exame preventivo, na detecção precoce e cura do câncer de colo uterino, a praticidade no agendamento e realização deste exame, a distribuição gratuita de preservativos na rede pública de saúde, o acompanhamento da mulher por profissionais capacitados e as campanhas preventivas visando a promoção da saúde da mulher.

Tendo em consideração os problemas de fluxo do SISCOLO, devido a amostra de 28 mulheres desse estudo ser subestimada, quando levamos em consideração amostras dos estudos tidos como referencial, sugere-se avaliação periódica realizada por sistemas oficiais de informação, visando aprimorar as informações e melhorar a qualidade e descrição das informações fornecidas.

Nesse sentido, essa pequena amostra de mulheres com NIC, entre 2009 a 2011, pode ser resultante de erros de coleta, acarretando em esfregaços cervicais sem células da endocérvice. Considera-se que um esfregaço ideal para o exame de Papanicolaou deve conter células da ectocérvice e endocérvice, pois esfregaços cervicais de portadoras de NIC apresentam mais células endocervicais alteradas do que aqueles de mulheres sem NIC. Outra possível explicação para a baixa incidência de NIC pode ser atribuído a possíveis erros de leitura dos esfregaços cervicais ${ }^{31}$, ou mesmo por falta de alimentação do banco de dados do SISCOLO.

\section{Conclusão}

A análise de dados do SISCOLO demonstrou que 28 mulheres foram acometidas com neoplasias intra-epiteliais cervicais entre os anos de 2009 a 2011, na cidade de Tangará da Serra-MT, sendo que a faixa etária de maior incidência está entre 30 a 39 anos e 50 a 59 anos, enaltecendo a importância do exame de Papanicolaou, independente da idade.

Os dados apresentados no presente trabalho demonstram uma redução no número de casos de neoplasias intra-epiteliais cervicais nos anos de 2010 e 2011, quando comparado com o ano de 2009, sugerindo um maior conhecimento das mulheres sobre o câncer de colo uterino, campanhas preventivas e a estrutura de atendimento fornecida pelo SUS.

Sendo assim, ressalta-se a importância da detecção precoce das lesões precursoras da cérvice, uma vez que, quando diagnosticadas nos estágios iniciais das neoplasias intra-epiteliais cervicais, aumentam-se as possibilidades de cura e evita-se a progressão da lesão para carcinoma invasivo.

Portanto, esse trabalho enfatiza o uso de método preservativo de barreira, pois mesmo não sendo uma barreira totalmente eficaz contra a infecção pelo HPV, fornece proteção, principalmente tendo em consideração o início precoce da atividade sexual e multiplicidade de parceiros, fatores esses que aumentam a contaminação.

\section{Agradecimentos}

A Secretaria de Saúde do Município de Tangará da Serra-MT. Ao Posto Central, em especial a Elizângela Ramos da Silva e Dra. Marinete de Sena e Silva. Ao Pólo Regional de Saúde de Tangará da Serra, principalmente a Matilde de Lima Perin. A Dra. Sabla Emmanuelle O. V. Santos pela contribuição científica.

\section{Referências}

1. Bittencourt NOS, Kliemann Neto JF. Rede social no sistema de saúde: um estudo das relações interorganizacionais em unidades de serviços de HIV/AIDS. Rev Adm Contem 2009; 13:87-104.

2. Doorbar J. Warts and all. mill hill essays. Londres: National Institute for Medical Research; 1999.

3. Carvalho NS. Bioética e doenças sexualmente transmissíveis. J Bras Doenças Sex Transm 2003;15(2):57-61.

4. Shope RE. Infectious papillomatosis of rabbits. J Exp Med 1933;58:607-25.

5. Strauss MJ, Shaw EW, Bunting H, Melnick J. "Crystalline" virus-like particles from skin papillomas characterized by intranuclear inclusion bodies. Exp Biol Med 1949;72(1):4650.

6. Galloway DA. Papillomavirus vaccines in clinical trials. Lancet Infec Dis 2003;3:469-75.

7. ZurHausen H. Viruses in human cancers. Science 1991;25:1167-73.

8. Focchi J. Papilomavirus humano e neoplasia genital. In: Piato S. Tratado de ginecologia. São Paulo: Artes Médicas; 2002.

9. Diógenes MAR, Varela ZMV, Barroso GT. Papillomavírus humano: repercussão na saúde da mulher no contexto familiar. Rev Gaúcha Enferm 2006;27(2):266-73.

10. Villa LL, Ault KA, Giuliano AR, Costa RLR, Petta CA, Andrade RP, et al. Immunologic responses following administration of a vaccine targeting human papillomavirus Types 6, 11, 16 and 18. Vaccine 2006;24:5571-83.

11. Instituto Nacional de Câncer (INCA). Neoplasia Intra-Epitelial Cervical - NIC. Ver Bras Cancerol 2000;46(4):355-7.

12. Crum CP. Aparelho genital feminino. In: Robbins SL, Cotran RS. Patologia, bases patológicas de doenças. Rio de Janeiro: Elsevier; 2005.

13. Marana HRC, Duarte G, Quintana SM. Fatores de risco para recidiva após tratamento de lesões provocadas pelo 
HPV no trato genital feminino. Rev Bras Ginecol Obstet 1999;21(4):201-5.

14. Souza NST, Melo VH, Castro LPF. Diagnóstico da infecção pelo HPV em Lesões do Colo do Útero em Mulheres HIV+: Acuidade da Histopatologia. Rev Bras Ginecol Obstet 2001;23(6):355-61.

15. Parellada CI, Pereyra EAG. Papilomaviroses humanas. In: Foccacia RV. Tratado de infectologia. São Paulo: Elsevier; 2005.

16. Figueirêdo PG, Derchain SFM, Sarian LOZ, Gontijo RC, Andrade LAA, Campos EA, et al. Detecção do DNA do papilomavírus humano após excisão da zona de transformação com alça diatérmica para tratamento de neoplasia intra-epitelial cervical. Rev Bras Ginecol Obstet 2003;25(1):9-15.

17. Castro-Vásquez MC, Arellano-Gálvez MC. Acesso a La informacíon de mujeres com VPH, displasia y câncer cervical in situ. Salud Pública Mex 2010;52:207-12.

18. Lawson MA. Human papillomavirus infection in adolescent and young women. Mo Med 2008;105(1):42-6.

19. Brasil. Ministério da Saúde. Portal da Saúde. 2005. [acesso em 6 ago 2014]. Disponível em http://portal.saude.gov.br/ saude/visualizar_texto.cfm?idtxt=22444.

20. Brasil. Ministério da Saúde.Departamento de Informações Estatísticas DATASUS. Brasília: Ministério da Saúde; 2011

21. Instituto Nacional de Câncer - INCA. Câncer de colo de útero. 2012. [acesso em 20 set 2014]. Disponível em http:// www.inca.gov.br/estimativa/2012/index.asp?ID $=5$.

22. Fonseca KCLE, Silveira LVPC, Pacheco JBP. Prevalência das neoplasias intra-epiteliais cervicais em mulheres atendidas pela unidade de ações e saúde da mulher e do adolescente da cidade de Anápolis, GO. Anuário Prod Inic Cient Disc 2010;13(17):151-63.
23. Uchimura NS, Nakano K, Nakano LCG, Uchimura TT. Qualidade e desempenho das colpocitologias na prevenção de câncer de colo uterino. Rev Assoc Med Bras 2009;55(5):56974.

24. Siqueira PLF, Leite SA, Toledo TY, Furtado TRP. Prevalência de carcinoma in situ em mulheres com neoplasia cervical intra-epitelial assistidas no município de Cacoal-RO. Rev Eletron Facimed 2011;3(3):344-52.

25. Nonnenmacher B, Breitenbach V, Villa LL, Prolla JC, Bozzetti MC. Identificação do papilomavírus humano por biologia molecular em mulheres assintomáticas. Rev Saúde Pública 2002;36(1):95-100.

26. Rama CH, Roteli-Martins CM, Derchain SFM, LongattoFilho A, Gontijo RC, Sarian LOZ, et al. Prevalência do HPV em mulheres rastreadas para o câncer cervical. Rev Saúde Pública 2008;42(1):123-30.

27. Fonseca AJ, Ferreira LP, Dalla-Benetta AC, Roldan CN, Ferreira MLS. Epidemiologia e impacto econômico do câncer de colo de útero no Estado de Roraima: a perspectiva do SUS. Rev Bras Ginecol Obstet 2010;32(8):386-92.

28. Taborda WC, Ferreira SC, Rodrigues D, Stávale JN, Baruzzi RG. Rastreamento do câncer de colo uterino em índias do Parque Indígena do Xingu, Brasil central. Rev Panam Salud Pública 2000;7(2):92-6.

29. Medeiros VCRD, Medeiros RC. Câncer de colo de útero: análise epidemiológica e citopatológica no Estado do Rio Grande do Norte. Rev Bras Anal Clin 2005;37(4):227-31.

30. Costa RF, Barros SMO. Prevalência de lesões intraepiteliais em atipias de significado indeterminado em um serviço público de referência para neoplasias cervicais. Acta Paul Enferm 2011;24(3):400-6.

31. Gompel C, Koss LG. Citologia ginecológica e suas bases anatomoclínicas. São Paulo: Manole; 1995. 